\title{
Diagnosis-Addison's Disease Secondary to Tuberculosis of the Adrenal Glands
}

\author{
Mrinal M. Patnaik, MD and Alaka K. Deshpande, MD
}

\begin{abstract}
A
diagnosis of Addison's disease secondary to tuberculosis of the adrenal glands was made for the case presented in the December 2007 Aperture. ${ }^{1}$ The computed tomography (CT) scan in figure 1 demonstrates the presence of free fluid in the patient's abdomen and bilateral cystic adrenal hyperplasia. The patient was started on four-drug anti-tuberculosis therapy (isoniazid, rifampin, pyrazinamide and ethambutol) along with hydrocortisone supplementation. Fludrocortisone was added to augment his blood pressure response. Over the next few weeks he showed a marked improvement in constitutional symptoms. The free fluid in the abdomen resolved, and he is currently on his eighth month of antituberculosis drug therapy, along with continued steroid supplementation.
\end{abstract}

In 1855, Thomas Addison described autopsy findings of six patients with adrenal tuberculosis, which continues to be one of the most common causes of adrenal insufficiency in the developing world. In a recent study Guo et $a,^{2}$ compared the contrast enhanced CT scan features of the adrenal glands in 42 patients with adrenal tuberculosis. Of these patients, $91 \%$ (38) had bilaterally enlarged adrenals and $51 \%(21)$ had enlarged glands with preservation of the contours. ${ }^{2}$ Other important, although less common causes of adrenal insufficiency that need to be considered in the clinical differential diagnosis include histoplasmosis, blastocmycosis, adrenal metastases, primary adrenal tumors and opportunistic infections related to HIV/AIDS. ${ }^{3}$

\section{References:}

1. Patnaik MM, Deshpande, AK. Cystic adrenal hyperplasia-diagnosis? Clin Med Res 2007;5:227

2. Guo YK, Yang ZG, Li Y, Ma ES, Deng YP, Min PQ, Yin LL, Hu J, Zhang XC, Chen TW. Addison's disease due to adrenal tuberculosis: Contrast-enhanced CT features and clinical duration correlation. Eur J Radiol 2006;62:126-131.

3. Oelkers W. Adrenal insufficiency. N Engl J Med 1996;335:1206-1212.

Author Affiliations: *Mrinal M Patnaik, MD, Department of Internal Medicine, University of Minnesota, Minneapolis, MN 554 I 4 USA, Email: mrinal.patnaik@gmail.com

Alaka Deshpande, MD, Department of Internal Medicine, Grant Medical College and Sir JJ Hospital, Mumbai, India

*Corresponding Author

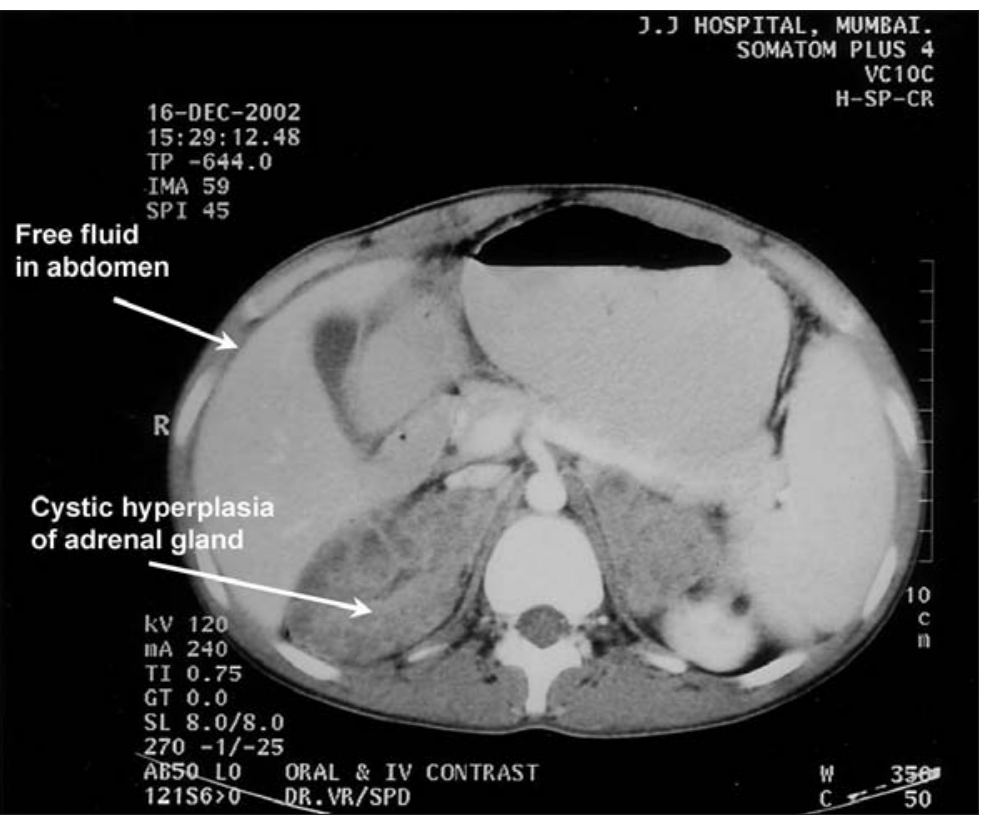

Figure 1. CT scan indicating bilateral cystic adrenal hyperplasia and free fluid in the abdomen (arrow points to the very fine, hypodense region between the liver margin and the anterior abdominal wall).

The Aperture, like the opening in the lens of a microscope that allows light to pass through, is a forum for art, humor, and images that provides a portal for new or different views of medicine and research. 